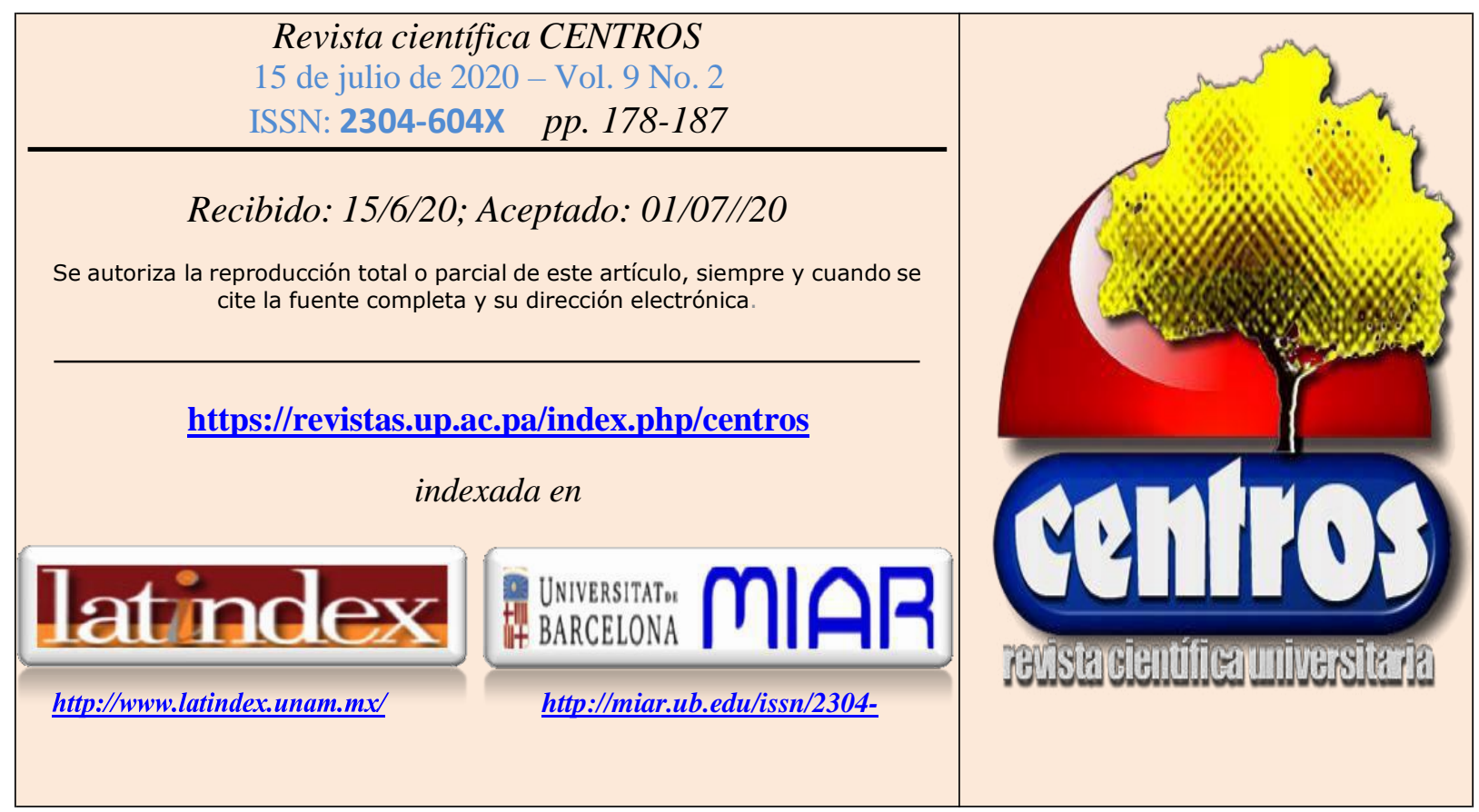

\title{
Alternativas de la Administración Educativa a nivel superior ante el efecto de la covid-19
}

\section{Alternatives of the Educational Management of the higher education before the effect of covid-19}

\author{
Marta S. de Peralta ${ }^{1}$ y Johana Marín ${ }^{1}$ \\ ${ }^{1}$ Universidad de Panamá. Centro Regional Universitario Panamá Este \\ martadeperalta@gmail.com
}

\section{Resumen}

Enfrentados a problemas inmediatos, los líderes universitarios necesitan, al mismo tiempo, proporcionar respuestas de emergencia para mantener a los estudiantes aprendiendo, y prepararse para las repercusiones de la crisis, reflexionando sobre las tendencias que pueden afectar a sus universidades: en cuanto al impacto que tendrá la crisis en la matrícula estudiantil, en el modelo de enseñanza y aprendizaje, en la relevancia de las actividades de investigación e innovación, y en el modelo de aportación de valor. Al hacerlo, reforzarán la importancia del liderazgo equipos rectorales, pero también directores de unidades académicas -, y mejorarán 
las oportunidades de fijar el rumbo en lugar de simplemente reaccionar ante los desafíos que se presentan en estos tiempos difíciles.

Palabras Claves: Pandemia, Educación Superior, Virtualidad, Administración Educativa, COVID-19.

\section{Abstract}

Facing immediate problems, university leaders need, to provide at the same time, emergency responses to keep students learning, and being prepared for the repercussions of the crisis, reflecting on the trends that may affect their universities as well: what impact will the crisis have on the student enrollment? What model of teaching and learning should be used to stimulate? how to increase the relevance of research and innovation activities? What is the value of the contribution model and other fundamental questions? In doing so, they will reinforce the importance of leadership, of the The university rectory, of the directors of academic units as well ; and the opportunities to set a course will be set forward rather than just reacting to the challenges of these difficult times.

Keywords: Pandemic, Higher Education, Virtuality, Educational Administration, Covid19.

\section{Introducción}

El mundo ha experimentado una pandemia producto del desarrollo del coronavirus (COVID-19). La globalización ha contribuido a la velocidad con la que el virus se ha propagado alrededor del planeta, lo cual ha provocado una paralización en todos los ámbitos -social, político y económico. Todavía no se han dimensionado los efectos de esta pandemia; sin embargo, se puede anticipar como una crisis de la cual es posible volver a una situación "normal" como la habíamos concebido anteriormente.

El efecto de esta pandemia también alcanza todos los niveles educativos, incluyendo la educación superior, lo que los obliga a actuar a la brevedad y acorde a las situaciones emergentes. Las instituciones de educación superior tienen un rol de importancia crítica en esta crisis; además de proveer importantes servicios. Las universidades continúan siendo una fuente fundamental de investigación enfocada en 
producir soluciones a problemas como el que el mundo enfrenta, por lo que deben ser las primeras en proponer acciones de solución que lidericen entornos de aprendizaje ininterrumpidos a todos los niveles sociales.

La inequidad social, antes y ahora, ha sido un desafío importante para el desarrollo de la educación superior, pero es tal vez más evidente durante la crisis actual. Una posible solución emerge de las Tecnologías de la Información y la Comunicación (TIC's); considerando que la población que atiende la educación superior corresponde al grupo denominado "generación millennials y posteriores", y para ellos la tecnología es más accesible y están más familiarizados con sus aplicaciones y usos. Según el BID 2020, el $41 \%$ de los millennials se dedica a estudiar, el $21 \%$ a trabajar, un $17 \%$ realiza ambas actividades y el $21 \%$ no tiene trabajo ni estudia. Casi ocho de cada diez jóvenes estudian, trabajan o hacen las dos.

Desde esta perspectiva, las instituciones de educación superior deben emprender todos los esfuerzos posibles para ofrecer cursos por tantos medios tecnológicos como sea posible, ya sean teléfonos celulares, tablets, computadoras, televisión, entre otras; con tal de maximizar el acceso al mayor número posible de estudiantes. (Sanz, Sáinz \& Capilla, 2020).

En el pasado, la mayoría de las instituciones de educación superior han actuado con lentitud en aprovechar el potencial de las nuevas tecnologías. Si se hubiese tenido una mayor apertura y flexibilidad al respecto, tal vez la transición hubiese sido menos disruptiva. En el futuro, la educación superior seguramente no será la misma que prevalecía antes de la pandemia, ya que las opciones no presenciales se convertirán en una alternativa más usada a partir de ahora. (Sánchez, 2020).

Los científicos han estado prediciendo, desde hace un buen tiempo, que una pandemia era inevitable, aunque no han tenido recursos financieros suficientes para desarrollar soluciones, particularmente en la educación superior, las instituciones de educación superior están en una situación que podría describirse bien por la metáfora de que dice "hay que reparar la bicicleta mientras vamos en ella". (UNESCO, 2020). 
Muchos políticos han optado por ignorar tales llamados de alerta. Es cierto que la política no ha causado esta crisis, pero es evidente que los políticos han contribuido a crear condiciones que propiciaron que hoy no estemos debidamente preparados. (UNESCO IESALC, 2020).

De hecho, se podría decir que la pandemia añade un grado más de complejidad crítica a una educación superior que, prácticamente en todo el mundo, pero en particular en la región de Latinoamérica, el Caribe y específicamente en Panamá, ya se enfrentaba a retos no resueltos como un crecimiento sin calidad, inequidades en el acceso y en los logros o la pérdida progresiva de financiamiento público. (UNESCO IESALC, 2020).

La situación es particularmente preocupante respecto de los estudiantes más vulnerables que ingresaron a la educación superior en condiciones más frágiles. Una disrupción en el entorno como la que está produciendo esta crisis puede convertir esa fragilidad en abandono reproduciendo así, una vez más, la exclusión a la que da lugar la inequidad que caracteriza el ingreso a la educación superior en la región. Esta inequidad se refleja igualmente en las elevadas tasas de abandono y no compleción de los estudios superiores: en promedio, solo la mitad de las personas entre 25 y 29 años que estaban matriculadas no completaron sus estudios, ya sea por abandono o porque aún continúan estudiando. De los que abandonan, la mitad lo hace en el primer año de su carrera (Ferreyra, Avitabile, Botero Álvarez, Haimovich Paz, \& Urzúa, 2017).

Siendo la educación no presencial la única opción en estos momentos, esta requiere de mayor disciplina y compromiso por parte del estudiante, lo que quizás explique que esta tenga o pueda tener más éxito entre los estudiantes de mayor edad, esto es, los de posgrado, frente a los de pregrado.

La pandemia del virus COVID-19 ha llevado a la Universidad de Panamá a tomar medidas tecnológicas, aplazar periodos de trámites, flexibilizar matrículas y ofrecer alternativas con el objetivo de que sus estudiantes no paralicen sus clases y educar a toda la comunidad. (Joaquín Villar, comm pers, 2020). 
En el caso de la Universidad de Panamá, desde 2010 desarrolla diversas estrategias de formación en entornos virtuales de aprendizaje para su planta docente; sin embargo, los datos reflejan poco interés de los docentes en estos cursos por no ser obligatorios ni estar regulada esta estrategia en la Universidad.

Actualmente, ante la situación del cierre de las instalaciones universitarias por los riesgos a la salud, la Universidad de Panamá a través del Consejo Académico, mediante el acuerdo Nro. 320 del 11 de marzo de 2020, estableció como medida de prevención contra la propagación del COVID-19 que todas las actividades administrativas, académicas y todas aquellas que signifiquen congregación de personas fueran suspendidas. Además, se determinó que el semestre académico se desarrollará de manera no presencial. En la Universidad de Panamá es la primera vez en que las clases se darán totalmente virtuales, para ello la institución dispone de siete plataformas digitales desde hace cinco años, pero debido a que las clases eran mayormente presenciales no se aprovechaba su uso. (Flores, 2020).

La Universidad de Panamá, actualmente cuenta con una matrícula de 75 mil estudiantes, e inició sus clases el 20 de abril de 2020. Por primera vez está efectuando el proceso de matrícula totalmente no presencial. (Zea, 2020).

También se contó con el apoyo de los proveedores de telefonía móvil del país (Movistar y Digitel), quienes ofrecieron acceso gratuito en sus respectivas redes de datos móviles, sin afectación en los planes de transferencia de datos individualmente contratados, todos los accesos y los servicios prestados desde la página web de la Universidad (up.ac.pa). Esta acción se emprendió en beneficio de toda la comunidad universitaria y en el estudiantado desde el proceso de inscripción hasta las clases virtuales. (UNESCO IESALC, 2020).

Con esta perspectiva en mente, se propone hacer una revisión de la situación actual de los hechos y proponer ideas para el fortalecimiento de estrategias de entornos virtuales de aprendizaje en la Universidad de Panamá. 


\section{Desarrollo}

En consecuencia, de lo mencionado anteriormente, todas las unidades académicas de la Universidad de Panamá se abocaron a realizar las tareas de atención de los estudiantes estableciendo dos opciones. En la primera dar una capacitación rápida a los docentes; y en la segunda darle libertad a sus docentes para que experimentaran cualquier opción válida de la educación no presencial.

Las respuestas fueron diversas $y$, en ese sentido, cabe destacar las experiencias de los Centros Regionales Universitarios de Panamá Este y Colón de la Universidad de Panamá, quienes tomaron la iniciativa de brindarle a los docentes una jornada de capacitación y actualización en Entornos Virtuales de Aprendizaje para la Educación Superior durante abril y mayo de los corrientes, el cual fue desarrollado con facilitadores nacionales y extranjeros, mediante herramientas tecnológicas aplicadas a los entornos virtuales de aprendizaje.

En el seminario participaron 195 docentes de diversas facultades tales como: Administración de Empresas y Contabilidad, Arquitectura y Diseño, Ciencias Agropecuarias, Ciencias Naturales, Exactas y Tecnologías, Informática Electrónica y Comunicación, Ingeniería, Administración Pública, Bellas Artes, Humanidades, Ciencias de la Educación, la mayoría con más de quince años de experiencia universitaria.

Un diagnóstico inicial de los participantes dio cuenta que la mayoría afirmaba que las estrategias de entornos virtuales de aprendizaje no eran apropiadas para la educación superior; poseían conocimientos básicos en programas como Microsoft Office (Word, Excel, Power Point) y pininos modestos con aplicaciones para diseño, grabación, transmisión y edición de videos e imágenes. Así mismo reconocían tener muy poco o nulo conocimiento sobre aplicaciones para el manejo de aulas virtuales. En cuanto al acceso a la tecnología se ponderó que la mayoría tenía como recursos de uso: celulares, computadoras, impresoras y acceso a internet constante.

Durante la capacitación teórica-práctica de 40 horas se abordaron elementos de adecuación curricular, creación de aulas virtuales, manejo de plataformas de comunicación y aplicaciones para crear ecosistemas de aprendizajes para las situaciones contextuales de los docentes y estudiantes. 
Al final de la capacitación se observó que los docentes lograron algunos cambios cualitativos que mencionaron en un foro grupal: manifestaron una aceptación oportuna hacia el uso de estrategias para los entornos virtuales de aprendizaje; estimaron que al ser la educación superior una actividad en evolución, no era productivo volver a los métodos tradicionales; consideraron que el trabajo colaborativo con los colegas y la participación de los estudiantes forman parte fundamental para el logro de los objetivos propuestos. También se manifestaron dispuestos a seguir capacitándose en entornos virtuales de aprendizaje hasta desarrollar un modelo particular para su ambiente de enseñanza-aprendizaje.

\section{Consideraciones Finales}

La Universidad de Panamá demostró liderazgo y asertividad al enfrentar la necesidad de atención para los estudiantes de todo el país mediante una opción moderna y adecuada a la situación caótica de Panamá y el mundo.

Los docentes de la Universidad de Panamá, a pesar de la escasa experiencia en entornos virtuales de aprendizaje aceptaron el reto y lo están llevando a cabo con un gradiente situacional delimitado por el conocimiento propio, la disponibilidad de conexión a la red, la disponibilidad de equipos, la falta de servicios básicos (luz y otros) y el recurso económico para comprar tiempo para conectarse adecuadamente para recibir las clases.

Cada docente se ha valido de la herramienta tecnológica que mejor domina, para llevar adelante sus actividades de enseñanza-aprendizaje. Es importante resaltar que, a pesar de las dificultades en este novedoso proceso, se ha observado que se cuenta con un número apreciable de asistencia estudiantil a las clases; los que emprenden el autoaprendizaje más allá de las propuestas dadas por los docentes en clases; se ha fomentado el trabajo en equipo para resolver los problemas planteados $y$, sobre todo, han disminuido considerablemente los costos de escolaridad en transporte y libros.

Ha quedado evidenciado que no hay respuestas perfectas ante esta emergencia; pero si se retrasan las acciones de revisión de estatutos, investigaciones sobre las mejores prácticas en la materia o se espera a que la comunidad discente se 
adapte a la enseñanza virtual; se estaría perdiendo un tiempo valioso para lograr una aproximación a las necesidades estudiantiles. Se deben ofrecer opciones viables en tanto que se logra un ajuste a una nueva realidad.

Las fórmulas tradicionales de educación no presencial, es decir, aquellas en las que el profesor sigue impartiendo una clase ordinaria que es retransmitida en directo y que puede ser recuperada en diferido, parecen ser las más apreciadas por los estudiantes porque son las que mejor reproducen la dinámica a la que están acostumbrados. Las iniciativas que intentan cambiar radicalmente las reglas de funcionamiento y exigen que los estudiantes salgan de su zona de confort sin ningún entrenamiento previo son menos apreciadas porque, por razones bien distintas, los estudiantes de pregrado tienden a ser más conservadores de lo que podría pensarse o estar menos preparados para cambiar de modalidad (Watts, 2016).

En cambio, el comportamiento de los estudiantes de posgrado parece ser, en este sentido, más abierto a metodologías participativas o que exigen un mayor grado de interacción entre ellos mismos y el profesorado.

La mayor parte de las inconformidades proviene de, que el contenido que se ofrece nunca fue diseñado en el marco de un curso de educación superior no presencial, sino que intenta paliar la ausencia de clases presenciales con clases virtuales sin mayor preparación previa.

A esto hay que añadir que las materias que buscan el desarrollo de competencias profesionales por medio de la práctica (clínicas, residencias pedagógicas, carreras de diseño, ingenierías, ciencias y, en general, todas aquellas fuertemente dependientes de talleres prácticos, trabajo en laboratorios o prácticas institucionales) generan mayor incertidumbre. También las matemáticas, en general, tienen más dificultades para hacer adaptaciones a lo virtual.

Los docentes que cuentan con un bagaje importante de experiencia en este ámbito, generada por ejemplo a través de programas de posgrado a distancia, y de los recursos digitales apropiados, probablemente no tengan grandes dificultades para garantizar la continuidad. En cualquier caso, no debe desestimarse que la curva de 
aprendizaje para la utilización eficiente de la tecnología en educación superior a distancia es muy pronunciada y requiere de apoyo externo en lo tecnológico y en lo pedagógico.

En los restantes casos de docentes, que son la mayoría, la opción más simple consiste en la transmisión de sesiones de clase por video, en directo o diferido. Y aquí es donde el profesorado puede ver la diferencia entre aquellas Instituciones de Educación Superior que ponen a su disposición herramientas y recursos como, por ejemplo, cursos de capacitación, y aquellas otras que no. (UNESCO IESALC, 2020).

Hay que aprovechar las ventajas de la educación no presencial como la posibilidad de personalizar la formación y reforzar los puntos débiles de cada estudiante. Además, reforzar la formación del profesorado en el uso de las metodologías no presenciales y su interacción en el aprendizaje son claves para el éxito. Tienen que saber cómo se hace la docencia en línea, aprender metodologías adecuadas, personalizar la docencia a sus alumnos, e incluso, crear sus propios recursos educativos. (Revista Nuve, 2020; Aljawarneh, 2019).

\section{Referencias Bibliográficas}

Aljawarneh, S. A. (2019). Reviewing and exploring innovative ubiquitous learning tools in higher education. Journal of Computing in Higher Education, 1-17

BID. (2020). Millennials, la generación incomprendida. Recuperado de: https://www.iadb.org/es/mejorandovidas/millennials-la-generacionincomprendida. Fecha: 14 de jun. de 2020.

Ferreyra, M. M., Avitabile, C., Botero Álvarez, J., Haimovich Paz, F., \& Urzúa, S. (2017). At a Crossroads: Higher Education in Latin America and the Caribbean. Washington, DC: World Bank.

Flores, E. (2020). Estrategias universitarias en los tiempos del Covid-19. Recuperado: https://www.prensa.com/sociedad/estrategias-universitariasen-los-tiempos-del-covid-19/ Fecha: 10 de junio de 2020.

IESALC (2020) Liderazgo en tiempos de COVID-19. Recuperado de http://www.iesalc.unesco.org/2020/04/30/liderazgo-en-tiempos-de-covid19/ Fecha: 14 de junio de 2020). 
REVISTA NUVE. (2020). El uso de Las TICS en el ámbito educativo. Recuperado de:https://www.revistanuve.com/el-uso-de-las-tics-en-el-ambitoeducativo/. Fecha: 10 de junio de 2020.

Sánchez, J. (2020). Educación virtual la pandemia que cambio la educación superior para siempre Recuperado:

https://www.eltiempo.com/vida/ciencia/educacion-virtual-la-pandemia-quecambio-la-educacion-superior-para-siempre-476390 Fecha: 10 de junio de 2020.

Sanz, I., Sáinz, J., \& Capilla, A. (2020). Efectos de la crisis del coronavirus en la educación. Madrid: Organización de Estados Iberoamericanos para la Educación, la Ciencia y la Cultura (OEI).

UNESCO. (2020). Crisis-sensitive educational planning. Paris: UNESCO.

UNESCO-IESALC (2020). COVID-19 y educación superior: De los efectos inmediatos al día después. Análisis de impactos, respuestas políticas y recomendaciones. Disponible en: http://www.iesalc.unesco.org/wpcontent/uploads/2020/04/COVID-19.pdf Fecha: 6 de abril de 2020,

Watts, L. (2016). Synchronous and asynchronous communication in distance learning: A review of the literature. Quarterly Review of Distance Education, 17(1), 23-32.

Zea, M. (2020). Estrategias universitarias en los tiempos del Covid-19. Recuperado: https://www.prensa.com/sociedad/estrategias-universitariasen-los-tiempos-del-covid-19/ Fecha: 9 de junio de 2020. 